\title{
Fatal retroperitoneal haemorrhage complicating anticoagulant therapy
}

\author{
G. D. O. LowE* \\ M.B., M.R.C.P. \\ J. H. MCKILLOP* \\ M.B., M.R.C.P. \\ A. G. PRENTICE $\dagger$
M.B., M.R.C.P.
*University Department of Medicine, Royal Infirmary, Glasgow G4 0SF, and
†University Department of Haematology, Western Infirmary, Glasgow G11 6NT
}

\begin{abstract}
Summary
Eight cases are reported of extensive retroperitoneal haemorrhage in patients receiving anticoagulant therapy who subsequently died and underwent postmortem examination. No patient had an obvious source of bleeding, and in 5 cases no other cause for death was apparent. Three patients had had vascular surgical procedures: the other 5 were given anticoagulants for suspected venous thrombo-embolism. Six cases presented as unexpected circulatory collapse with rapid demise. Four patients had in addition gastro-intestinal bleeding of varying degree, with no obvious source. This acute massive type of retroperitoneal bleeding contrasts with the commoner presentations of pain, swelling, bruising and compression neuropathy: a high index of suspicion and urgent treatment are required if mortality is to be reduced.
\end{abstract}

\section{Introduction}

Spontaneous retroperitoneal haemorrhage is an unusual but well recognized occurrence in patients receiving anticoagulant therapy (Curry and Bacon, 1974) as well as in haemophiliacs (van Trotsenburg, 1975). Three recent series have suggested that retroperitoneal bleeding in patients receiving intravenous heparin is commoner than previously recognized, the incidence being 4.3-6.6\% (UrokinasePulmonary Embolism Trial, 1973; Curry and Bacon, 1974; Mant et al., 1977). Reports of fatal cases are, however, rare (Flanc, Kakkar and Clarke, 1968; Curry and Bacon, 1974). Recent personal experience of 2 patients who died from retroperitoneal haemorrhage while receiving anticoagulants prompted the authors to survey the recent records of the 5 major Glasgow general hospitals for further cases with post-mortem documentation.

\section{Methods}

Case records and post-mortem reports for all

Correspondence and requests for reprints: Dr G. D. O. Lowe, Royal Infirmary, Glasgow G4 OSF. patients with a coding of retroperitoneal haemorrhage, from 1970 to 1976 inclusive, were obtained from the 5 major teaching hospitals in Glasgow. Each hospital has adult general medical and surgical wards in addition to specialist units. Details were selected of patients treated with anticoagulants during their final illness in whom there was post-mortem confirmation of a large retroperitoneal haemorrhage which was thought to be the sole cause of death or a major contributory cause.

\section{Case reports}

Six cases were obtained from the survey, and cases were already known to the authors.

\section{Case 1}

A 38-year-old man with mitral incompetence underwent mitral valve replacement. Bilateral thoracotomy was performed with cardiopulmonary bypass, using a femoral artery for return of arterial blood. Intravenous heparin $(21000 \mathrm{u}$.) and protamine $(420 \mathrm{mg})$ were administered during bypass; the operation was uneventful. On return to the Intensive Care Unit he became shocked with falling central venous pressure. Chest radiograph was unremarkable and there was minimal pleural drainage. He was returned to theatre the same day to exclude cardiac tamponade. On opening the chest the pericardial cavity was empty and there was little blood in the heart; cardiac arrest occurred and resuscitation was unsuccessful. Post-mortem examination revealed a large retroperitoneal haematoma and also abundant blood clot in the peritoneal cavity, without an apparent source of bleeding. The mitral valve prosthesis was satisfactory and there was no blood in either pleural cavity.

\section{Case 2}

A 55-year-old man was admitted to hospital for 10 days for an uncomplicated anterior myocardial infarction. Five days after discharge he was reacinitted with acute ischaemia of both lower limbs 
and absent femoral pulses. Bilateral iliofemoral embolectomy was performed and had to be repeated twice within $24 \mathrm{hr}$ owing to recurrent arterial occlusion. Cross-matching of blood was initially impossible owing to gross rouleaux formation: further tests revealed a free, cold auto-antibody of large thermal amplitude. Corticosteroids, i.v. dextran, and i.v. heparin were commenced. A fourth embolectomy was required for left femoral occlusion 3 days later, but thereafter the circulation to the legs and the patient's general condition were satisfactory. Histology of the emboli showed recent thrombus. There was no evidence of recurrent myocardial infarction. Heparin was given by intravenous infusion for 7 days in doses of 30000-80000 u./ $24 \mathrm{hr}$ (mean $60000 \mathrm{u} . / 24 \mathrm{hr}$ ) without monitoring. Warfarin was then given for 7 days: Thrombotest was performed on 5 days and was less than $5 \%$ of normal on one day, and between 5 and $10 \%$ on the other occasions. On the seventh day of warfarin therapy there was a sudden, unspecified deterioration in his condition with rapid demise. At postmortem a large retroperitoneal haemorrhage was found: no source of bleeding was seen. There was a recent left coronary thrombus and corresponding recent anterior myocardial infarction, left ventricular aneurysm, and mural thrombus. No significant atheroma of lower limb vessels was apparent.

\section{Case 3}

A 67-year-old woman underwent phenol block injection of the left lumbar sympathetic chain for intermittent claudication in the left leg of recent onset. Next day both radial pulses became impalpable and blood pressure was unrecordable in both arms. Three days later all leg pulses below both femorals became impalpable. Arch aortography showed bilateral subclavian artery occlusion. Intravenous infusion of heparin was commenced (40 $000 \mathrm{u} . / 24 \mathrm{hr}$ ) without monitoring. Five days later she developed central abdominal pain, had a small haematemesis, and became shocked. Heparin was reduced to $30000 \mathrm{u} . / 24 \mathrm{hr}$, and pethidine and chlorpromazine administered. She died $12 \mathrm{hr}$ later. At post-mortem examination a massive retroperitoneal haematoma was found, without obvious source. Both subclavian and both common iliac arteries were stenosed, with recent thrombus in the left subclavian. Histology of these vessels showed a giant-cell arteritis.

\section{Case 4}

A 64-year-old man was admitted to hospital with pleuritic chest pain and a painful, swollen right leg. The clinical picture of ilio-femoral venous thrombosis was confirmed by ascending venography.
Intravenous heparin infusion (40000 u./24 hr) was given for 14 days. Whole blood clotting time was determined daily and ranged from 10-12 min. From the tenth day he complained of intermittent left loin pain. Warfarin was given in addition to heparin over the next 3 days (total $30 \mathrm{mg}$ ); Thrombotest was not performed. On the fourteenth day he suddenly collapsed with shock, abdominal pain and ileus. He was treated with intravenous fluids, blood and vitamin $\mathrm{K}$, but died $8 \mathrm{hr}$ later. At post-mortem, organizing right iliofemoral venous thrombosis and a minor pulmonary embolus were confirmed. There was a large left-sided retroperitoneal haemorrhage without obvious source. An in situ carcinoma of the right kidney was found, but this was discrete from the haemorrhage and the left kidney was normal.

\section{Case 5}

A 68-year-old male was admitted with cardiac failure and chest pain. There was no evidence of acute myocardial infarction. Four days later he complained of pleuritic chest pain: chest radiograph was normal and a clinical diagnosis of pulmonary embolism was made. Intravenous infusion of heparin (40 $000 \mathrm{u} . / 24 \mathrm{hr})$ was commenced. Whole blood clotting time was 10 min the next day but was not repeated. On the fourth day of heparin therapy he suddenly developed pain in the abdomen and right loin with paralytic ileus and anaemia. Heparin was stopped and intravenous plasma given, but massive gastro-intestinal bleeding occurred and he died after $30 \mathrm{~min}$. Post-mortem confirmed a large retroperitoneal haematoma and blood in the gastro-intestinal tract, with no obvious source. There was no evidence of venous thromboembolism.

\section{Case 6}

A 72-year-old man was admitted to hospital with a painful, swollen left calf. On ascending venography there was no filling of the left anterior or posterior tibial veins. Intravenous heparin infusion (40000 u./ $24 \mathrm{hr}$ ) was started on admission. On the second day whole blood clotting time was $8.5 \mathrm{~min}$ and heparin was increased to $50000 \mathrm{u} . / 24 \mathrm{hr}$ : clotting time next day was $10 \mathrm{~min}$. On the seventh day he developed pleuritic chest pain, sinus tachycardia and right bundle branch block with a normal chest radiograph. Whole blood clotting time was $10 \mathrm{~min}$. Heparin was increased to $60000 \mathrm{u} . / 24 \mathrm{hr}$. repeat clotting time was $12 \mathrm{~min}$. Next day he developed abdominal pain and tenderness, paralytic ileus, and shock. Heparin was stopped and intravenous protamine, fluids, plasma and blood administered, but he died $8 \mathrm{hr}$ later. Post-mortem confirmed massive retroperitoneal haemorrhage without obvious source. There was no evidence of venous thromboembolism. 


\section{Case 7}

A 70-year-old woman was admitted semi-conscious in severe biventricular failure with cardiomegaly, left lower lobe consolidation, left bundle branch block, hypoxaemia and metabolic acidosis. Pulmonary embolism was diagnosed and she received oxygen, digoxin, diuretics, sodium bicarbonate and intravenous heparin infusion (40000 u./ $24 \mathrm{hr}$ ) without monitoring. Her circulatory state and acidosis improved. Two days after admission urine and faeces both contained occult blood on routine testing. Next day she suddenly became shocked. Whole blood clotting time was greater than $50 \mathrm{~min}$, and her haematocrit had fallen from 0.43 on admission to $0 \cdot 27$. Heparin was stopped and intravenous protamine $(60 \mathrm{mg})$ given, but she died shortly afterwards. At post-mortem there was a large retroperitoneal haematoma without apparent source. Examination of the heart showed biventricular hypertrophy with normal valves and coronary arteries; there was no evidence of venous thrombosis, pulmonary embolus or pneumonia.

\section{Case 8}

A 59-year-old woman with mitral stenosis, on warfarin for 6 years, experienced abdominal pain after twisting her trunk in a fall. Next day she was given co-trimoxazole for a coincidental sore throat, but this was stopped after 3 days because of vomiting and worsening abdominal pain. On admission after another 3 days there was guarding and fullness in the right abdomen and loin. Thrombotest was less than $5 \%$, haemoglobin $10 \mathrm{~g} / \mathrm{dl}$, and blood urea $25 \mathrm{mmol} / \mathrm{l}$. Warfarin was continued and co-trimoxazole restarted. Next day she developed atrial fibrillation, hypotension and oliguria: intraabdominal bleeding was suspected. Warfarin and co-trimoxazole were stopped, and intravenous vitamin $\mathrm{K}(20 \mathrm{mg})$, gentamicin, lincomycin and plasma were given. On the third day her cardiac failure was worse and she was transferred to the Intensive Therapy Unit, where parenteral digoxin, frusemide and fluids were administered, with central venous pressure monitoring. A diuresis ensued but renal failure was established and her blood urea rose steadily. Intermittent positive pressure ventilation was required from the sixth day. Her right-sided abdominal signs persisted, and progressive anaemia was corrected with packed cells. A persistent pyrexia and neutrophilia from the fourth day did not respond to gentamicin, lincomycin and ampicillin. Platelet count was never less than $70 \times 10^{9} / 1$. On the fifth day pulmonary embolism was suspected on clinical grounds and intravenous heparin infusion (24 000 u./24 hr) started. Next day her partial thromboplastin time was 108 seconds (control $42 \mathrm{sec}$ ). On the ninth day she developed melaena; whole blood clotting time was $8 \mathrm{~min}$ and heparin was stopped. On the tenth day her blood urea was $83 \mathrm{mmol} / \mathrm{l}$ and peritoneal dialysis was commenced: following aspiration of blood from the trachea she developed a tension pneumothorax and died. At post-mortem there was total collapse of the right lung with a tension pneumothorax and mitral stenosis. A massive retroperitoneal haematoma, with no obvious source of blood loss, was centred round the right kidney and was compressing its artery. Histology of the right kidney showed tubular necrosis: the left kidney was normal.

\section{Discussion}

Eight patients receiving anticoagulant therapy have been described who developed extensive retroperitoneal haemorrhage which was verified at postmortem and believed to contribute to death. Only a few isolated cases of this fatal complication have been hitherto reported (Flanc et al., 1968; Curry and Bacon, 1974). In 5 patients of the present series retroperitoneal haemorrhage appeared to be the sole cause of death. In case 5, simultaneous heparin-related bleeding into the gastrointestinal tract probably contributed to death. The manner of demise in case 2 was not apparent from the records and fatal cardiac dysrhythmia cannot be excluded. Case 8 was a complicated one, and the immediate cause of death was probably a tension pneumos thorax, but it appears that retroperitoneal haemore rhage initiated the chain of events, promoting renat and cardiac failure as well as contributing to death in its own right.

Three patients developed retroperitoneal haemorrhage after vascular procedures-femoral artery cannulation for cardiopulmonary bypass in case 1 , repeated embolectomy in case 2 , and phenol sympathetic block in case 3 (who also had a giant-cell arteritis). However, no evidence of leakage from major blood vessels was apparent at post-mortem. The other 5 patients were anticoagulated for suspected venous thromboembolism: again no source of retroperitoneal bleeding was evident at post-mortem. Venous thromboembolism was confirmed at post-mortem in only one patient, which illustrates the problem of diagnosis of venous thrombosis and pulmonary embolism.

Anticoagulant-related retroperitoneal haemorrhage has been reviewed by Curry and Bacon (1974). Pain and tenderness in the abdomen, loin or groin is the usual presentation: a palpable mass or bruising may develop in these areas, and compression neuropathy or paralytic ileus can occur. Two patients in the present series (cases 4 and 8 ) complained of pain in the abdomen or loin for several days before they became acutely ill, and it is possible that earlier diagnosis and reversal of anticoagulation might 
have prevented further bleeding in these instances. In the other 6 patients the presentation was sudden and death rapid, the normal picture being hypovolaemic shock with or without abdominal pain or paralytic ileus. Four patients had in addition revealed gastrointestinal bleeding of some degree: in no case was a source for this found at post-mortem. It is possible that excessive anticoagulation causes bleeding from multiple small blood vessels in both retroperitoneal tissues (Curry and Bacon, 1974) and the gut wall.

One patient was heparinized only during cardiac bypass surgery, and appeared to develop retroperitoneal and intraperitoneal haemorrhage at the end of operation, despite protamine correction. The other 7 patients received continuous intravenous infusions of heparin for 3-14 days (mean 6.5 days) in average daily doses of 24000-60000 u. (mean $42000 \mathrm{u}$.). This mean daily dose is higher than that required to maintain the whole blood clotting time or activated partial thromboplastin time within a 'therapeutic' range in 3 reported series (O'Sullivan et al., 1968; Basu et al., 1972; Mant et al., 1977). In only 2 patients was regular monitoring of clotting times performed. Whether or not monitoring of heparin infusion reduces bleeding complications is unknown. In the 2 patients in whom whole blood clotting time was monitored it was never excessively prolonged: the range of 10-12 min in these patients would be regarded by several authors as indicating inadequate doses of heparin. However, as there is some evidence for an association between prolonged clotting times and bleeding (O'Sullivan et al., 1968; Urokinase-Pulmonary Embolism Trial, 1973) detection of excessive anticoagulant effect (as was belatedly discovered in case 7) and its correction might prevent at least some haemorrhages. Haemorrhage did not become manifest until after several days of heparin. The authors' clinical impression that the risk of all types of bleeding complication increases after the first 2 days of heparin treatment is in agreement with that of Mant et al. (1977). Possible reasons for this might be reduction in the anti-heparin activity of blood which is present in the acute stages of thrombosis (Dewhurst and Poller, 1965), and the thrombocytopenia which may develop after several days' heparin infusion (Bell et al., 1976). Three patients also received warfarin: monitoring was not performed in one case, and revealed excessive anticoagulant effect (thrombotest less than $5 \%$ ) in the other 2 on at least one day. In 2 patients drug interactions may have increased the effects of anticoagulants - concurrent administration of dextran and heparin in case 2, and co-trimoxazole and warfarin in case 8 .

Although retroperitoneal haemorrhage is an unusual cause of death in patients on anticoagulants, it may be more frequent than previously noted. It was not possible for the authors to ascertain the total incidence of fatal retroperitoneal bleeding in patients receiving heparin infusions in Glasgow during the study period. Sudden circulatory collapse might be misinterpreted as a recurrence of the primary problem for which anticoagulants were given, for example pulmonary embolism, and unless postmortem examination is carried out the true cause of death may be missed. The true incidence of fatal retroperitoneal haemorrhage might thus be underestimated. Recent reports suggest that the incidence of all forms of retroperitoneal haemorrhage in patients receiving heparin infusions is about $5 \%$ (Urokinase-Pulmonary Embolism Trial, 1973; Curry and Bacon, 1974; Mant et al., 1977). Increased awareness of this potentially lethal complication may lead to earlier diagnosis, earlier treatment, and reduced mortality.

\section{Acknowledgments}

We thank Dr I. Hodsman and the records department staff in the Glasgow hospitals studied (Royal Infirmary, Western Infirmary, Victoria Infirmary, Stobhill Hospital, and Southern General Hospital) for their assistance.

\section{References}

Basu, D., Gallus, A., Hirsh, J. \& Cade, J. (1972) A prospective study of the value of monitoring heparin treatment with the activated partial thromboplastin time. New England Journal of Medicine, 187, 324.

Bell, W.R., Tomasulo, P.A., Alving, B.M. \& Duffy, T.P (1976) Thrombocytopenia occurring during the administration of heparin. Annals of Internal Medicine, 85, 155.

CurRY, P.V.L. \& BACON, P.A. (1974) Retroperitoneal haemorrhage and neuropathy complicating anticoagulant therapy. Postgraduate Medical Journal, 50, 37.

DeWhuRst, F. \& Poller, L. (1965) Antiheparin activity of some human blood protein fractions and their possible relationship to thrombosis. Journal of Clinical Pathology, 18, 339.

Flanc, C., Kakkar, V.V. \& Clarke, M.B. (1968) The detection of venous thrombosis of the legs using ${ }^{125}$ I-labelled fibrinogen. British Journal of Surgery, 55, 742.

Mant, M.J., O'Brien, B.D., Thong, K.L., Hammond, G.W., BiRTWhistle, R.V. \& GRACE, M.G. (1977) Haemorrhagic complications of heparin therapy. Lancet, $\mathbf{i}, 1133$.

O'Sullivan, E.F., Hirsh, J., McCarthy, R.A. \& DE GRUCHY, G.C. (1968) Heparin in the treatment of venous thromboembolic disease: administration, control and results. Medical Journal of Australia, 2, 153.

URokinase-PulmoNARY Embolism Trial (1973) Circulation, 47 (Suppl. 2), 1.

van Trotsenburg, L. (1975) In: Handbook of Hemophilia (Ed. by Brinkhous, K.M. \& Hemker, H.C.), p. 398. Excerpta Medica, Amsterdam. 